\title{
Research Article \\ Retractions and Homomorphisms on Some Operations of Graphs
}

\author{
M. Abu-Saleem (iD \\ Department of Mathematics, Faculty of Science, Al-Balqa Applied University, Salt 19117, Jordan \\ Correspondence should be addressed to M. Abu-Saleem; mohammedabusaleem2005@yahoo.com
}

Received 18 May 2018; Revised 9 August 2018; Accepted 13 September 2018; Published 1 October 2018

Academic Editor: Francisco Balibrea

Copyright (c) 2018 M. Abu-Saleem. This is an open access article distributed under the Creative Commons Attribution License, which permits unrestricted use, distribution, and reproduction in any medium, provided the original work is properly cited.

\begin{abstract}
The aim of the present article is to introduce and study a new type of operations on graph, namely, edge graph. The relation between the homomorphisms and retractions on edge graphs is deduced. The limit retractions on the edge graphs are presented. Retractions on a finite number of edge graphs are obtained.
\end{abstract}

\section{Introduction and Preliminaries}

Graph theory is rapidly moving into the mainstream of mathematics. The prospects of further development in algebraic graph theory and important link with computational theory indicate the possibility of the subject quickly emerging at the forefront of mathematics. Its scientific and engineering applications, especially to communication science, computer technology, and system theory, have already been accorded a place of pride in applied mathematics. Graphs serve as mathematical models to analyze successfully many concrete real-world problems. A certain problem in physics, chemistry, genetics, psychology, sociology, and linguistics can be formulated as problems in graph theory. Also, many branches of mathematics such as game theory, group theory, matrix theory, probability, and topology have interactions with graph theory. Some puzzles and various problems of a practical nature have been instrumental in the development of various topics in graph theory. The theory of acyclic graphs was developed for solving problems of electrical networks and the study of trees was developed for enumerating isomers of organic compounds. This paper describes the operation of a graph from the viewpoint of an identification [1-10].

A graph is an ordered $G=(V(G), E(G))$, where $V(G) \neq \phi$, $E(G)$ is a set disjoint from $V(G)$, elements of $V(G)$ are called the vertices of $G$, and elements of $E(G)$ are called the edges. A graph is connected if, for every partition of its vertex set into two nonempty sets $X$ and $Y$, there is an edge with one end in
$X$ and one end in $Y$; otherwise, the graph is disconnected. A graph $H$ is said to be a subgraph of a graph $G$ if $V(H) \subseteq V(G)$ and $E(H) \subseteq E(G)$. A graph in which each pair of distinct vertices is adjacent is called a complete graph. A complete graph with $\mathrm{n}$ vertices is denoted by $K_{n}$ [11]. The chromatic number $\chi(G)$ of a graph $G$ is the minimum number of colors required for proper vertex coloring of $G$. A $m$-coloring of a graph $G$ is a vertex coloring of $G$ that uses at most $m$-colors. A graph $G$ is said to be $m$-colorable if $G$ admits a proper vertex coloring using at most $m$ colors [11]. Let $G$ and $H$ be two graphs. A function $\phi: V(G) \longrightarrow V(H)$ is a homomorphism from $G$ to $H$ if it preserves edges, that is, if for any edge $[u, v]$ of $G,[\phi(u), \phi(v)]$ is an edge of $H[12]$. A retract of a graph $G$ is a subgraph $H$ of $G$ such that there exists a homomorphism $r: G \longrightarrow H$, called retraction with $r(x)=x$ for any vertex $x$ of $H$ [7]. A core is a graph which does not retract to a proper subgraph [12].

\section{The Main Results}

Aiming at our study, we will introduce the following.

Definition 1. Let $G_{1}$ and $G_{2}$ be two connected graphs, where $e_{1}$ is an edge of $G_{1}, e_{2}$ is an edge of $G_{2}$, and $G_{1} \cap G_{2}=\phi$; then we define the edge graph $G_{1} \underline{\vee} G_{2}$ by gluing together the two edges $e_{1}$ and $e_{2}$.

Theorem 2. Let $G_{1}$ and $G_{2}$ be two connected graphs. Then $\chi\left(G_{1} \underline{\vee}_{2}\right)=\max \left\{\chi\left(G_{1}\right), \chi\left(G_{2}\right)\right\}$. 
Proof. Let $\chi\left(G_{1} \underline{\vee} G_{2}\right)=m$. At that point, there exists an $m$-coloring $\omega$ of $G_{1} \underline{\vee} G_{2}$. Since $\omega$ assigns different colors to every two adjacent vertices of $G_{1}$ and $G_{2}, G_{1}$ and $G_{2}$ are $m$ colorable and so $\chi\left(G_{1}\right) \leq \chi\left(G_{1} \underline{\vee} G_{2}\right)$ and $\chi\left(G_{2}\right) \leq \chi\left(G_{1} \underline{\vee} G_{2}\right)$. Also, using symmetry $\chi\left(G_{1}\right) \geq \chi\left(G_{2}\right)$. Beginning with an ideal coloring of $\chi\left(G_{1}\right)$, we can incorporate an ideal coloring of $\chi\left(G_{2}\right)$ by exchanging a pair of color names to make the coloring agree at two vertices of common edge graphs. This produces a proper coloring of $G_{1} \underline{\vee} G_{2}$.

Theorem 3. The graphs $G_{1}$ and $G_{2}$ are subgraphs of $G_{1} \vee G_{2}$. Also, for any tree $G_{1}$ and $G_{2}$, the graph $G_{1} \underline{V}_{2}$ is also a tree.

Proof. The proof of this theorem is clear.

Theorem 4. Suppose that $G_{1}, G_{2}, \ldots, G_{n}$ are connected graphs; then there is a sequence of nontrivial retractions $\left\{r_{k}\right.$ : $\left.\underline{\vee}_{i=1}^{n} G_{i} \longrightarrow \underline{\vee}_{i=1}^{n} G_{i}, k=1,2 \ldots n\right\}$, where $\underline{\vee}_{i=1}^{n} G_{i}$ are glued along the same edge such that $r_{k}\left(\underline{\vee}_{i=1}^{n} G_{i}\right)$ is a proper subgraph of $\underline{\mathrm{V}}_{i=1}^{n} G_{i}$.

Proof. Let $r_{1}: \underline{\vee}_{i=1}^{n} G_{i} \longrightarrow \underline{\bigvee}_{i=1}^{n} G_{i}$ be a retraction from $\underline{\vee}_{i=1}^{n} G_{i}$ into itself and $r_{1}\left(\underline{\vee}_{i=1}^{n} G_{i}\right)=G_{1} \underline{\vee} G_{2} \underline{\vee} \ldots \underline{\vee} \underline{r}_{1}\left(G_{s}\right) \ldots \underline{\vee} G_{n}$ for $s=1,2, \ldots, n$. Since $r_{1}\left(G_{s}\right)$ is a proper subgraph of $G_{s}$, it follows that $r_{1}\left(\underline{\vee}_{i=1}^{n} G_{i}\right)$ is a proper subgraph of $\underline{\mathrm{V}}_{i=1}^{n} G_{i}$. Also, if $r_{2}\left(\underline{\vee}_{i=1}^{n} G_{i}\right)=G_{1} \underline{\vee} G_{2} \underline{\vee} \cdots \underline{\vee} r_{2}\left(G_{s}\right) \underline{\vee} \cdots \underline{\vee} r_{2}\left(G_{k}\right) \underline{\vee} \cdots \underline{\vee} G_{n}$ for $k=1,2, \ldots, n, s<k$ and $r_{2}\left(G_{s}\right), r_{2}\left(G_{k}\right)$ are subgraphs of $G_{s}, G_{k}$, respectively, then $r_{2}\left(\underline{\vee}_{i=1}^{n} G_{i}\right)$ is a proper subgraph of $\underline{\vee}_{i=1}^{n} G_{i}$. Moreover, by continuing this process if $r_{n}\left(\underline{\vee}_{i=1}^{n} G_{i}\right)=$ $\underline{\mathrm{V}}_{i=1}^{n} r_{n}\left(G_{i}\right)$, then $r_{k}\left(\underline{\mathrm{V}}_{i=1}^{n} G_{i}\right)$ is a proper subgraph of $\underline{\mathrm{V}}_{i=1}^{n} G_{i}$.

Theorem 5. Let $G_{1}$ and $G_{2}$ be two graphs; then there is a homomorphism $f: G_{1} \longrightarrow G_{2}$ iff $G_{2}$ is a retract of $G_{1} \underline{\vee} G_{2}$.

Proof. Let $f: G_{1} \longrightarrow G_{2}$ be a homomorphism. Since $G_{2}$ is subgraph of $G_{1} \underline{\vee} G_{2}$, then there exists a homomorphism $r: G_{1} \underline{\vee} G_{2} \longrightarrow G_{2}$ with $r(x)=x$, for any vertex $x$ of $G_{2}$ and so $G_{2}$ is a retract of $G_{1} \vee G_{2}$. Conversely, assume that $G_{2}$ is a retract of $G_{1} \underline{\vee} G_{2}$; thus $r: G_{1} \underline{\vee} G_{2} \longrightarrow G_{2}$ is a homomorphism with $r(x)=x$ for any vertex $x$ of $G_{2}$, and so there is a homomorphism $f: G_{1} \longrightarrow G_{2}$.

Theorem 6. Let $G_{1}$ and $G_{2}$ be connected graphs; then $K_{n}$ is a retract of graph $G_{1}$ or $G_{2}$, iff $K_{n}$ retract of $G_{1} \underline{\vee} G_{2}$.

Proof. Suppose $G_{1}$ and $G_{2}$ are connected graphs and $K_{n}$ is a retract of graph $G_{1}$ or $G_{2}$. Then there is a homomorphism $r_{1}: G_{1} \longrightarrow K_{n}$ such that $r_{1}(x)=x$, or a homomorphism $r_{2}: G_{2} \longrightarrow K_{n}$ such that $r_{2}(x)=x$, for any vertex $x$ of $K_{n}$. Since $K_{n}$ is a core and it is subgraph of $G_{1}$ or $G_{2}$, it follows that $K_{n}$ is subgraph of $G_{1} \underline{\vee} G_{2}$ and so there is a homomorphism $r: G_{1} \underline{V}_{2} \longrightarrow K_{n}$ such that $r(x)=x$, for any vertex $x$ of $K_{n}$. Conversely, suppose $K_{n}$ is a retract $G_{1} \underline{\vee} G_{2}$; then $K_{n}$ is subgraph of $G_{1} \underline{\vee} G_{2}$ and $K_{n} \underline{\vee} K_{n}$ and so $K_{n}$ is a retract of graphs $G_{1}$ or $G_{2}$.

Theorem 7. Let $T$ be any tree of size $n$; then there is a sequence of nontrivial retractions $\left\{r_{i}, i=1,2, \ldots n\right\}$ such that $\lim _{n \rightarrow \infty} r_{n}\left(r_{n-1}\right) \ldots\left(r_{1}(T)=K_{2}\right.$.

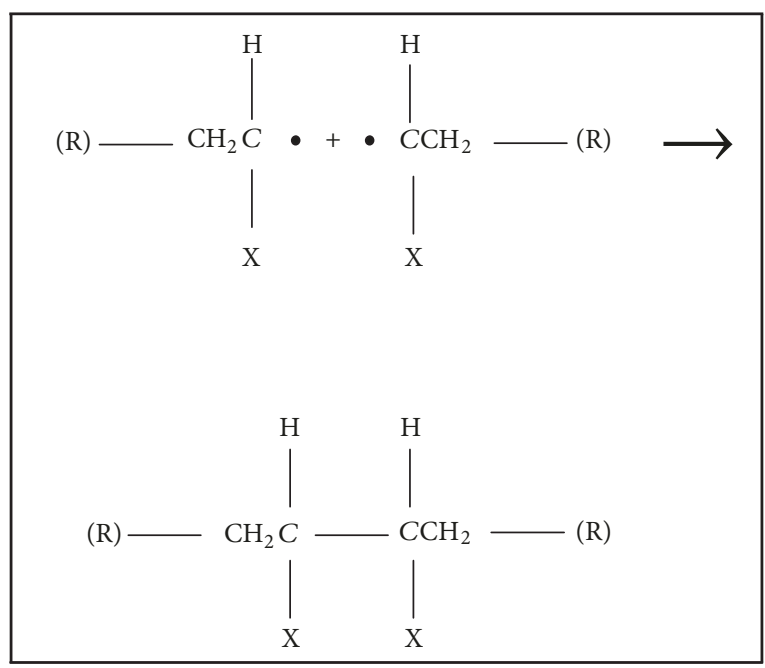

Figure 1

Proof. Consider the following sequence of retractions:

$r_{1}: T \longrightarrow T_{1}$ is nontrivial retraction, where $T_{1}$ is subgraph of $T$ and $1 \leq \operatorname{Size}\left(T_{1}\right) \leq n-1$,

$r_{2}: r_{1}(T) \longrightarrow r_{1}\left(T_{1}\right)$, where $r_{1}\left(T_{1}\right)$ is subgraph of $r_{1}(T)$ and $1 \leq \operatorname{Size}\left(r_{1}\left(T_{1}\right)\right) \leq n-2$,

$r_{n}: r_{n-1}\left(r_{n-2}\right) \ldots\left(r_{1}(T) \longrightarrow r_{n-1}\left(r_{n-2}\right) \ldots\left(r_{1}\left(T_{1}\right)\right.\right.$, where $r_{n-1}\left(r_{n-2}\right) \ldots\left(r_{1}\left(T_{1}\right)\right.$ is subgraph of $r_{n-1}\left(r_{n-2}\right) \ldots\left(r_{1}(T)\right.$, and $\lim _{n \rightarrow \infty} r_{n}\left(r_{n-1}\right) \ldots\left(r_{1}(T)\right.$ is a tree of size 1. Therefore, $\lim _{n \rightarrow \infty} r_{n}\left(r_{n-1}\right) \ldots\left(r_{1}(T)=k_{2}\right.$.

Theorem 8. Suppose that $G_{1}$ and $G_{2}$ are connected graphs; then $\left.\lim _{n \longrightarrow \infty} r_{n}\left(G_{1} \underline{\vee} G_{2}\right)\right)=\lim _{n \longrightarrow \infty} r_{n}\left(G_{1}\right) \underline{\operatorname{vim}} \lim _{n \rightarrow \infty} r_{n}\left(G_{1}\right)$.

Proof. If $G_{1}$ and $G_{2}$ are connected graphs, then we get the following induced subgraphs $\left.\lim _{n \rightarrow \infty} r_{n}\left(G_{1} \underline{\vee} G_{2}\right)\right), \lim _{n \longrightarrow \infty} r_{n}\left(G_{1}\right), \lim _{n \rightarrow \infty} r_{n}\left(G_{1}\right)$

and each of them is isomorphic to $k_{2}$. Since, $k_{2} \approx k_{2} \underline{\vee} k_{2}$, it follows that $\left.\lim _{n \longrightarrow \infty} r_{n}\left(G_{1} \underline{\vee} G_{2}\right)\right)=$ $\lim _{n \rightarrow \infty} r_{n}\left(G_{1}\right) \underline{\operatorname{vim}} \lim _{n \rightarrow \infty} r_{n}\left(G_{1}\right)$.

\section{Some Applications in Chemistry and Biology}

(i) A polymer is composed of many repeating units called monomers. Starch, cellulose, and proteins are natural polymers. Nylon and polyethylene are synthetic polymers. Polymerization is the process of joining monomers. Polymers may be formed by addition polymerization and one basic step in addition polymerization is combination as in Figure 1, which occurs when the polymer's growth is stopped by free electrons from two growing chains that join and form a single chain. The following diagram depicts combination, with the symbol (R) representing the rest of the chain. This is a representation type of connected two graphs into an edge graph.

(ii) Peptide bonds constitute the representation of an edge graph by linking two amino acids as in Figure 2, which is a 


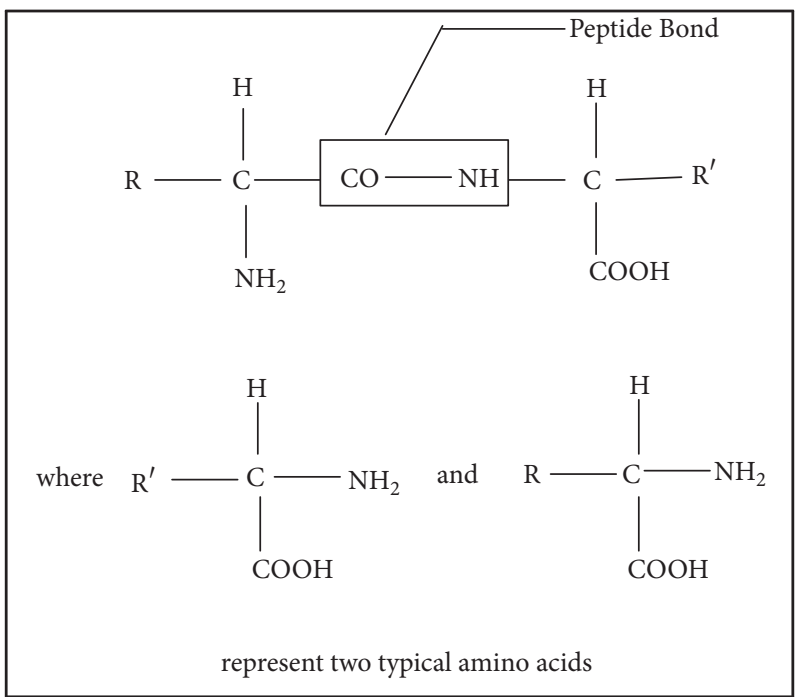

FIGURE 2
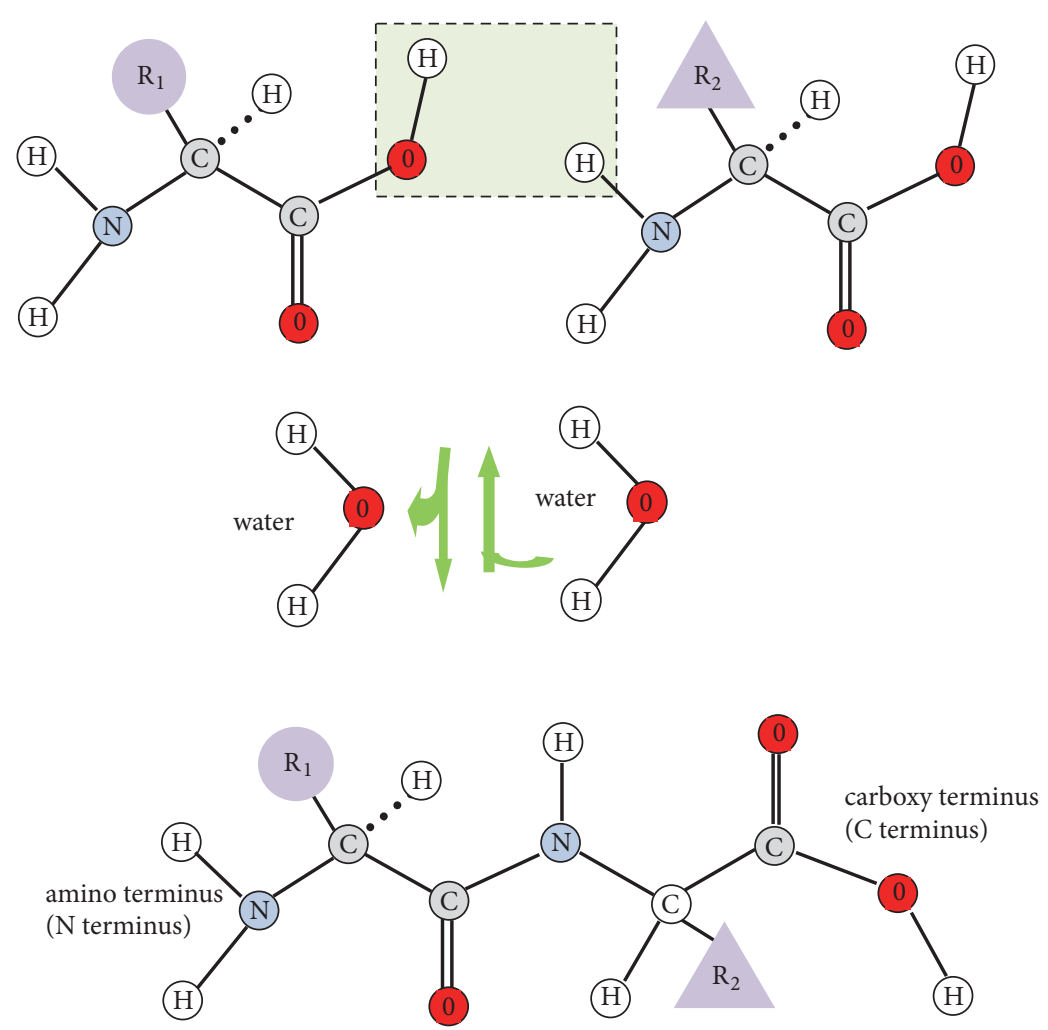

FiguRE 3

representation graph of connected two typical amino acids into an edge graph.

In Figure 3, peptide pond and formation hydrolysis: Formation (top to bottom) and hydrolysis from bottom to top of a peptide bonds require conceptually loss and addition, respectively, of a molecule of water. The actual chemical synthesis and hydrolysis of peptide bonds in the cell are enzymatically controlled processes that in the synthesis nearly always occur on the ribosome and are directed by an
mRNA template. The end of a polypeptide with the free of amino group is known as the amino terminus ( $\mathrm{N}$ terminus) and with the free carboxyl group is the carboxyl terminus (C terminus). This is a representation of connected two graphs into an edge graph.

\section{Data Availability}

No data were used to support this study. 


\section{Conflicts of Interest}

The author declares that they have no conflicts of interest.

\section{References}

[1] M. Abu-Saleem, "Folding on the wedge sum of graphs and their fundamental group," APPS. Applied Sciences, vol. 12, pp. 14-19, 2010.

[2] M. Abu-Saleem, "Dynamical manifold and their fundamental group," Advanced Studies in Contemporary Mathematics, vol. 20, no. 1, pp. 125-131, 2010.

[3] M. Abu-Saleem, "Conditional fractional folding of a manifold and their fundamental group," Advanced Studies in Contemporary Mathematics, vol. 20, no. 2, pp. 271-277, 2010.

[4] M. Abu-Saleem, "On dynamical chaotic de Sitter spaces and their deformation retracts," Proceedings of the Jangjeon Mathematical Society. Memoirs of the Jangjeon Mathematical Society, vol. 14, no. 2, pp. 231-238, 2011.

[5] M. Abu-Saleem, "On the dynamical hyperbolic 3-spaces and their deformation retracts," Proceedings of the Jangjeon Mathematical Society. Memoirs of the Jangjeon Mathematical Society, vol. 15, no. 2, pp. 189-193, 2012.

[6] M. Abu-Saleem, "On chaotic homotopy group," Advanced Studies in Contemporary Mathematics, vol. 23, no. 1, pp. 69-75, 2013.

[7] P. Hell and J. Nešetřil, Graphs and Homomorphisms, vol. 28, Oxford University Press, Oxford, UK, 2004.

[8] S. Pirzada and A. Dharwadker, "Applications of Graph Theory," J.KSIAM, vol. 11, no. 4, pp. 19-38, 2007.

[9] A. T. White, Graphs, Groups and Surfaces, North-Holland Publishing Co., Amsterdam, Holland, 1973.

[10] R. J. Wilson and J. J. Watkins, Graphs, An Introductory Approuch, A First Course in Discrete Mahematics, Canada John Wiley and sons, Inc., 1990.

[11] G. Chartrand and P. Zhang, Chromatic Graph Theory, Taylor and Francis group, 2009.

[12] G. Hahn and C. Tardif, "Graph homomorphisms: structure and symmetry," in Graph symmetry, vol. 497 of NATO ASI Series C 497, pp. 107-166, Kluwer, 1997. 


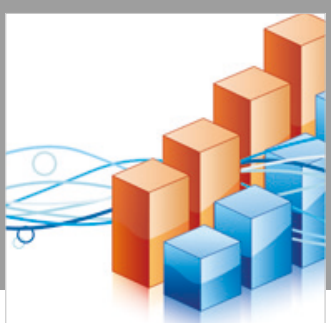

Advances in

Operations Research

\section{-n-m}
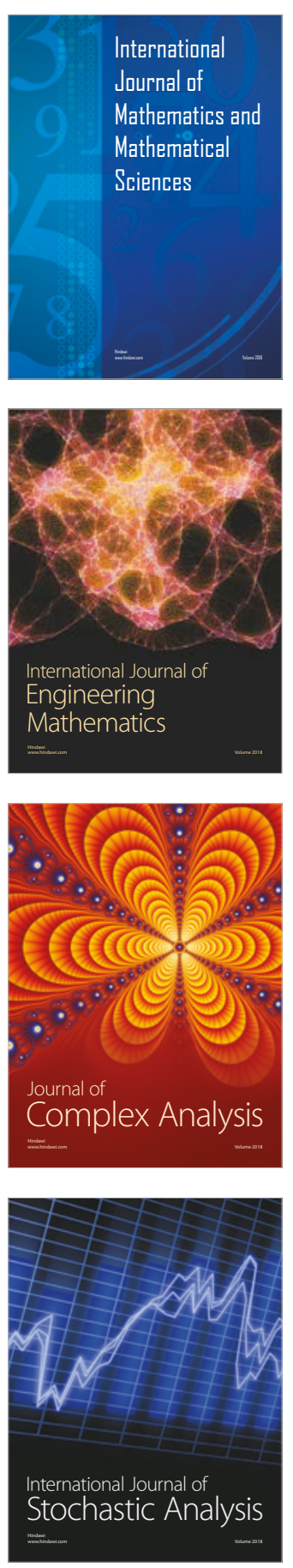
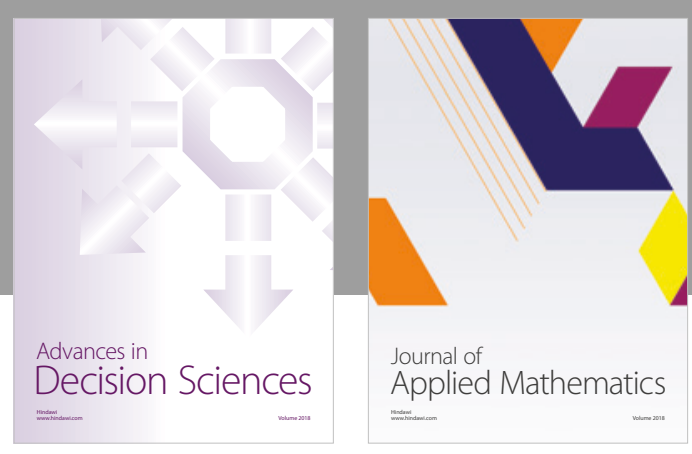

Journal of

Applied Mathematics
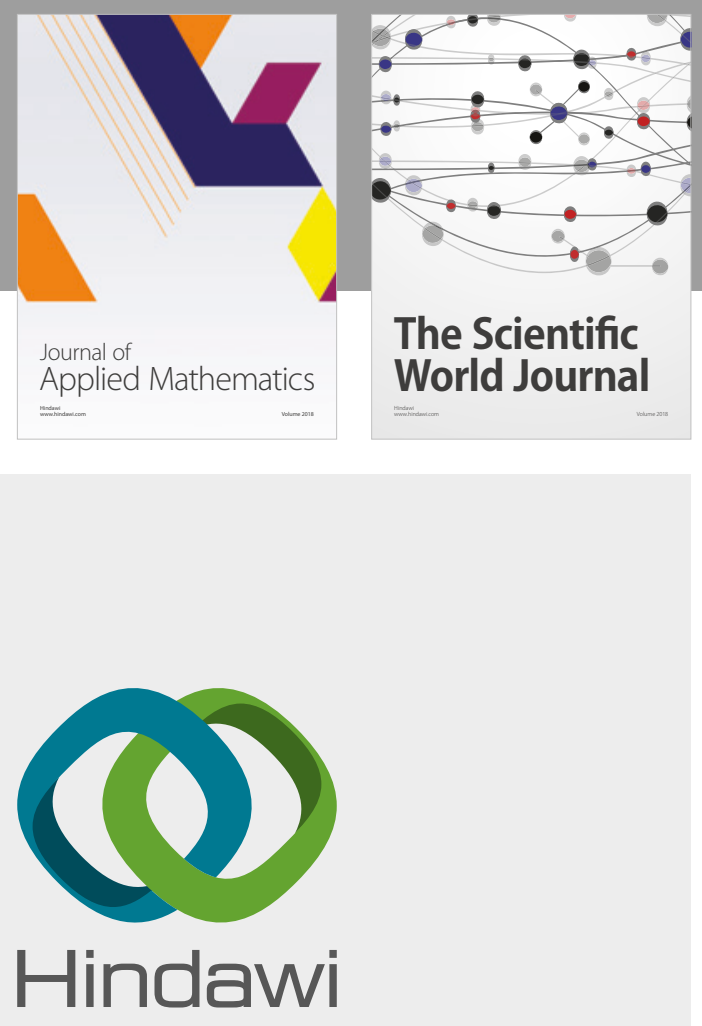

Submit your manuscripts at

www.hindawi.com

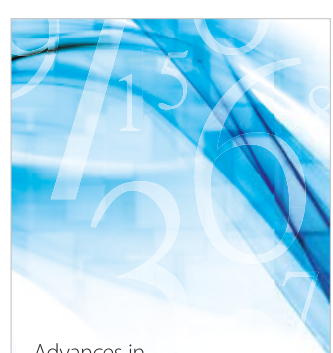

Advances in
Numerical Analysis
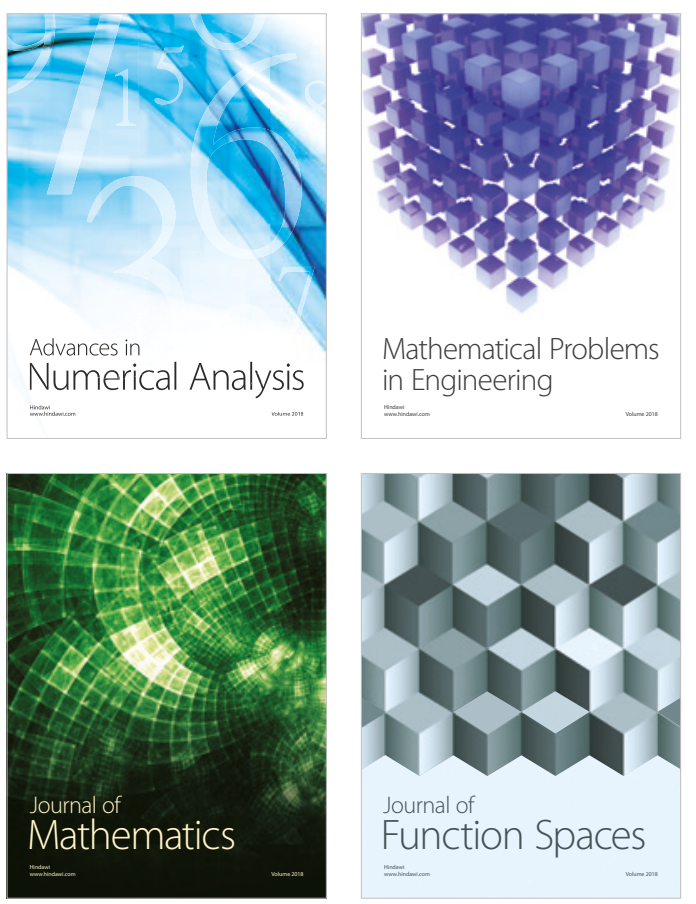

Mathematical Problems in Engineering

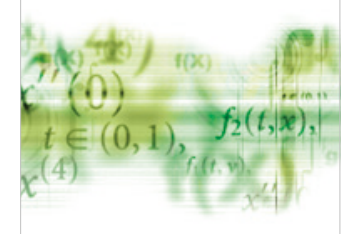

International Journal of

Differential Equations

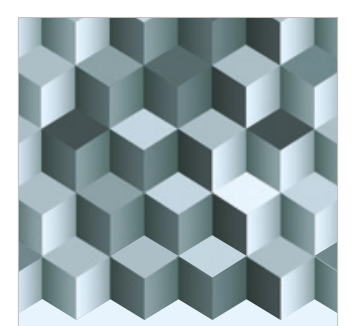

Journal of

Function Spaces

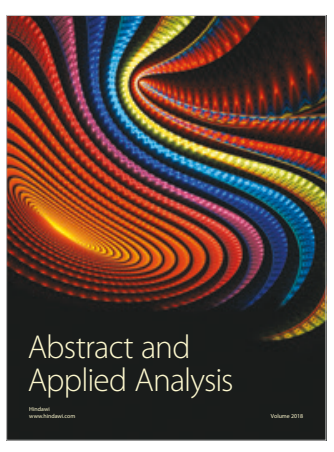

The Scientific

World Journal

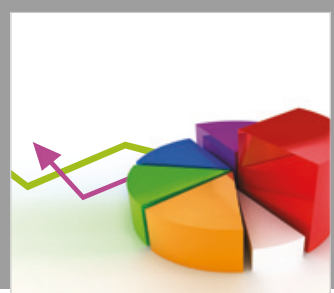

Journal of

Probability and Statistics
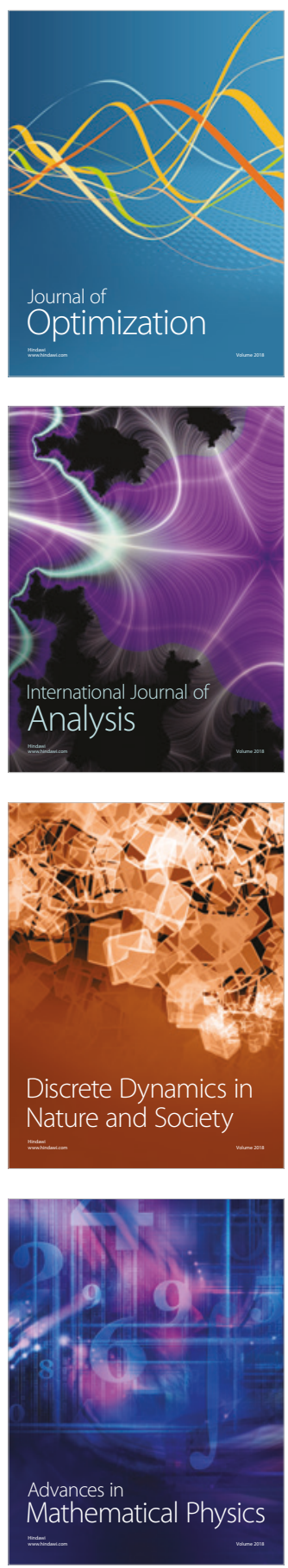